\title{
Robust stability for delayed port-Hamiltonian systems using improved Wirtinger-based inequality ${ }^{\star}$
}

\author{
Said Aoues ${ }^{1}$, Warody Lombardi ${ }^{2}$, Damien Eberard ${ }^{1}$ and Alexandre Seuret ${ }^{3}$
}

\begin{abstract}
This paper addresses robust stability issues of interconnected port-Hamiltonian systems with polytopic uncertainty and time-varying delay. On the basis of a LyapunovKrasovskii functional and the Wirtinger's inequality (known to be less conservative than the popular Jensen's inequality) we show the improvements of the newly proposed criterion with respect to other existing ones. The stability analysis is derived based on a delay independent criterion. A classical nonlinear example taken from the literature illustrates the relevance of the results.
\end{abstract}

\section{INTRODUCTION}

Modeling complex nonlinear systems can be tackled by a network representation of energy exchanges between interconnected subsystems. This is the underlying idea of portHamiltonian systems (PHSs) [12]. A fundamental property of this class of systems concerns their composability: interconnection of PHSs results in a PHS where the overall energy equals the sum of the subsystems energies. PHSs are also known to satisfy a passive equality, which is employed to conclude on stability by picking the energy (storage) function as a Lyapunov candidate. However, the picture is different when introducing delay in the interconnection structure: the interconnected system is no longer Hamiltonian and therefore the seek of other Lyapunov candidates is mandatory.

Classically, stability of time-delay systems is addressed from a state space representation. The dynamics includes the present and the past history of the trajectories as well [10]. Stability analysis is a challenging issue since delays may cause unstable closed-loop behavior [7].

Current efforts have been made to achieve robust stability for time-delay systems [2], firstly deriving delay-independent conditions [6], [8] and secondly delay-dependent conditions [1], [5], [13], in the form of a feasibility problem under Linear Matrix Inequalities (LMI) constraints.

To the best of the authors' knowledge, the literature regarding the stability of time-delay port-Hamiltonian systems

\footnotetext{
${ }^{1}$ Said Aoues and Damien Eberard are with AMPERE Lab, UMR CNRS 5005, INSA Lyon, 25 Av. Jean Capelle, 69621 Villeurbanne, France. E-mail: said.aoues, damien.eberard @insa-lyon.fr

2 Warody Lombardi is with CEA-LETI, Minatec Campus, 17 rue des Martyrs, 38054 Grenoble Cedex, France. E-mail: warody. lombardiecea.fr

${ }^{3}$ Alexandre Seuret is with CNRS, LAAS, 7 avenue du colonel Roche, F-31400 Toulouse, France and Univ de Toulouse, LAAS, F-31400 Toulouse, France. E-mail:a seuretelaas . fr

${ }^{\star}$ This work is supported by the French National Research Agency through the ANR project entitled Approximation of Infinite Dimensional Systems

${ }^{\star}$ This work is also partially supported by the French National Research Agency through the ANR project entitled LimICoS, contract number 12 BS03 00501
}

is weak. In [14], the stability of a class of time-delay Hamiltonian systems was investigated while some new results on generalized Hamiltonian realization were proposed. The stability conditions of the interconnected port-Hamiltonian systems using the negative feedback interconnection have been studied using Lyapounov-Krasovskii candidates, constructed from the Hamiltonian [9]. By means of a modified LyapunovKrasovskii candidate and adding equality constraints, a less conservative condition is given in [15]. The generalization of these results is given in [4], where the delays are assumed to be unknown time-varying functions. Notice that all these results are based on Jensen inequality to establish the stability criterion.

In contrast with the literature, we investigate the robust stability of the interconnected port-Hamiltonian systems with polytopic uncertainty using the recently appeared Wirtingerbased integral inequality [11]. The main advantage remains in the fact that one can use the same Lyapunov-Krasovskii functional, without any additional constraints. We will show that this inequality also allows the derivation of delaydependent stability conditions for delayed port-Hamiltonian systems. The sufficient condition is expressed in terms of LMIs, and is shown to be less conservative than other methods based on Jensen inequality. Then, the criterion is adapted to the robust stability analysis of polytopic systems. In this case, we will provide a delay-independent stability condition. To conclude, a numerical example is processed to show the relevance of the proposed method.

The paper is organized as follows: section II recalls the class of port-Hamiltonian systems and the feedback interconnection property. Delay interconnection of PHSs is presented section III. In Section IV, a delay-dependent stability condition is introduced and robust stability with polytopic system is derived. The particular case of delayindependent stability conditions is also treated in the section V. A numerical example is studied in Section VI and shows relevance of the proposed results. Section VII draws the concluding remarks.

Notations: Throughout the paper $\mathbb{R}^{n}$ denotes the $n$-dimensional Euclidean space with inner product $\langle\cdot, \cdot\rangle$. The notation $\mathcal{Q}>0$ (or $\mathcal{Q}<0$ ) means that the matrix $\mathcal{Q}$ is symmetric positive (or negative) definite. In symmetric block matrices, an asterisk $(*)$ represents a term that is induced by symmetry. $\nabla_{X}$ and $\nabla_{X}^{2}$ stand for the gradient and hessian operators respectively. The notation $[\cdot]_{t}$ (or simply with the index ${ }_{t}$ ) indicates that $[\cdot]$ is taken at time $t$, opposite to $[\cdot]_{t-h(t)}$ for instance. 


\section{PORT-HAMILTONIAN FRAMEWORK}

Let us first recall some properties inherent in the portHamiltonian formulation of dynamical systems.

\section{A. Port-Hamiltonian systems}

Throughout the paper, we shall consider nonlinear portHamiltonian systems with dissipation, whose dynamics are described by the following equations [12]:

$$
(\Sigma):\left\{\begin{array}{l}
\dot{x}=(J(x)-R(x))\left[\nabla_{x} H\right]_{t}+G(x) u, \\
y=G(x)^{T}\left[\nabla_{x} H\right]_{t},
\end{array}\right.
$$

where $x \in \mathbb{R}^{n}$ is the state (or Hamiltonian variables) vector and $J(x)$ is a skew-symmetric matrix called the structure matrix. Note that $J(x)$ is full rank and satisfies $J(x)+$ $J(x)^{T}=0 . R(x)$ is the positive semi-definite damping matrix $\left(R(x)=R(x)^{T} \geqslant 0\right)$. The matrix $G(x) \in \mathbb{R}^{n \times m}$ represents the input force matrix and $G(x) u$ denotes the generalized forces resulting from the control inputs $u \in \mathbb{R}^{m} . y \in \mathbb{R}^{m}$ is the system output. The output vector $y$ for this class of systems is imposed by the input vector field $G(x)$. Also, we say that $y$ is conjugate to $u$. To simplify the calculations in this paper, we consider that the matrices $J, R$ and $G$ are constant. Remind that $\left[\nabla_{x} H\right]_{t}$ denotes the gradient of $H$ with respect to $x$ and considered at time $t$.

Computing the time variation of $H$ along trajectories of (1) and integrating from $t_{0}$ to $t>t_{0}$ leads to the following energy balance equation:

$$
H(x(t))=H\left(x\left(t_{0}\right)\right)+\int_{t_{0}}^{t} y^{T}(s) u(s) \mathrm{d} s-\int_{t_{0}}^{t}\left[\nabla_{x} H\right]_{s}^{T} R\left[\nabla_{x} H\right]_{s} \mathrm{~d} s .
$$

From the dissipative theory framework, $H$ is a storage function and $y^{T} u$ is a supply rate with unit power. Hence, since $H$ is bounded from below, system (1) is said to be passive as it is dissipative w.r.t the supply rate $y^{T} u$. Equation (2) also reads as follows: the amount of energy of the system at time $t$ equals the amount of energy at time $t_{0}$ increased by the energy supplied through the port variables (positively or negatively) and decreased by the dissipated energy. Hence, it is worth noting that stability of the unforced system (1) can be immediately analyzed by taking $H$ as Lyapunov candidate, since $R>0$ and (2) reduces to $\dot{H}<0$, then the system (1) is asymptotically stable in the sense of Lyapunov.

\section{B. Interconnection of port-Hamiltonian systems}

Consider now two port-Hamiltonian systems given by:

$$
\left(\Sigma_{i}\right):\left\{\begin{array}{l}
\dot{x}_{i}=\left(J_{i}-R_{i}\right)\left[\nabla_{x_{i}} H_{i}\right]_{t}+G_{i} u_{i} \\
y_{i}=G_{i}^{T}\left[\nabla_{x_{1}} H_{i}\right]_{t}
\end{array}\right.
$$

It is well-known that port-Hamiltonian systems are composable, i.e. by considering two port-Hamiltonian systems $\Sigma_{1}$ and $\Sigma_{2}$, their interconnection through a power-conserving structure yields a dynamical system $\Sigma_{12}$ which again is a port-Hamiltonian system: the Hamiltonian framework is preserved under interconnection.

By denoting $\left(u_{1}, y_{1}\right)$ the port variables of system $\Sigma_{1}$, with state $x_{1}$, and $\left(u_{2}, y_{2}\right)$ the port variables of system $\Sigma_{2}$ with state $x_{2}$, one can consider the feedback interconnection:

$$
\left[\begin{array}{l}
u_{1} \\
u_{2}
\end{array}\right]=\left[\begin{array}{cc}
0 & -\mathrm{I} \\
\mathrm{I} & 0
\end{array}\right]\left[\begin{array}{l}
y_{1} \\
y_{2}
\end{array}\right]
$$

as drawn in Fig. 1.

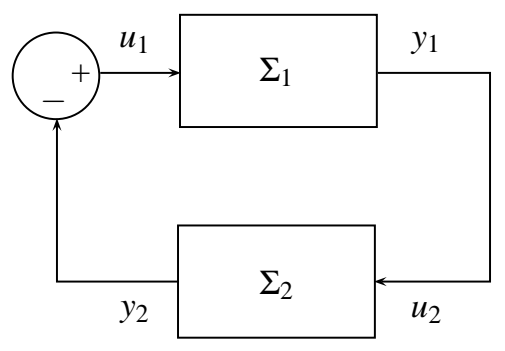

Fig. 1. Feedback interconnection of two port-Hamiltonian systems.

Then, the dynamics of the interconnected system $\Sigma_{12}$ is written as:

$$
\begin{aligned}
\frac{\mathrm{d}}{\mathrm{d} t}\left[\begin{array}{l}
x_{1} \\
x_{2}
\end{array}\right] & =\left[\begin{array}{cc}
J_{1}-R_{1} & -G_{1} G_{2}^{T} \\
G_{2} G_{1}^{T} & J_{2}-R_{2}
\end{array}\right]\left[\begin{array}{l}
{\left[\nabla_{x_{1}} H_{1}\right]_{t}} \\
{\left[\nabla_{x_{2}} H_{2}\right]_{t}}
\end{array}\right] \\
\dot{\mathcal{X}} & =(\mathcal{J}-\mathcal{R})\left[\nabla_{\mathcal{X}} \mathcal{H}\right]_{t},
\end{aligned}
$$

where

$$
\mathcal{X}=\left[\begin{array}{l}
x_{1} \\
x_{2}
\end{array}\right], \mathcal{J}=\left[\begin{array}{cc}
J_{1} & -G_{1} G_{2}^{T} \\
G_{2} G_{1}^{T} & J_{2}
\end{array}\right], \mathcal{R}=\left[\begin{array}{cc}
R_{1} & 0 \\
0 & R_{2}
\end{array}\right] .
$$

The total energy $\mathcal{H}$ of $\Sigma_{12}$ equals the sum of the subsystems energies, i.e. $\mathcal{H}=H_{1}+H_{2}, \mathcal{J}$ gather the skew-symmetric part and $\mathcal{R}$ the damping part. It is then easy to see that the derivative of $\mathcal{H}$ along the trajectories of (5) is given by:

$$
\frac{\mathrm{d} \mathcal{H}_{t}}{\mathrm{~d} t}:=\left\langle\nabla_{\mathcal{X}} \mathcal{H}_{t}, \frac{\mathrm{d} \mathcal{X}_{t}}{\mathrm{~d} t}\right\rangle=-\nabla_{\mathcal{X}} \mathcal{H}_{t}^{T} \mathcal{R} \nabla_{\mathcal{X}} \mathcal{H}_{t} \leq 0,
$$

showing that $\Sigma_{12}$ is stable since, using the same argument as previously, $\mathcal{H}$ is a Lyapunov candidate. Asymptotic stability is achieved whenever $\mathcal{R}>0$.

For the sake of clarifying the main idea and simplifying the presentation, we only detail here the full interconnection case. However, partial interconnection, which means that a subset of port variables are constrained by interconnection, processes in the same way.

Unfortunately, in most cases, this interconnection will not be perfectly achieved as in (4). In [9] and [15], the authors showed that the structure and the stability properties of the interconnected systems, obtained with negative feedback interconnection between two port-Hamiltonian systems, can be affected by the presence of time-delays. Therefore, the stability analysis can be assessed by the application of the Lyapunov-Krasovskii theorem, as we will show latter on in this article.

\section{PROBLEM FORMULATION AND PRELIMINARIES}

Contrarily to the interconnection case described in (4), time-varying delays must be taken into account in a more realistic framework. The feedback interconnection becomes:

$$
\left[\begin{array}{l}
{\left[u_{1}\right]_{t}} \\
{\left[u_{2}\right]_{t}}
\end{array}\right]=\left[\begin{array}{cc}
0 & -\mathrm{I} \\
\mathrm{I} & 0
\end{array}\right]\left[\begin{array}{l}
{\left[y_{1}\right]_{t-h(t)}} \\
{\left[y_{2}\right]_{t-h(t)}}
\end{array}\right],
$$




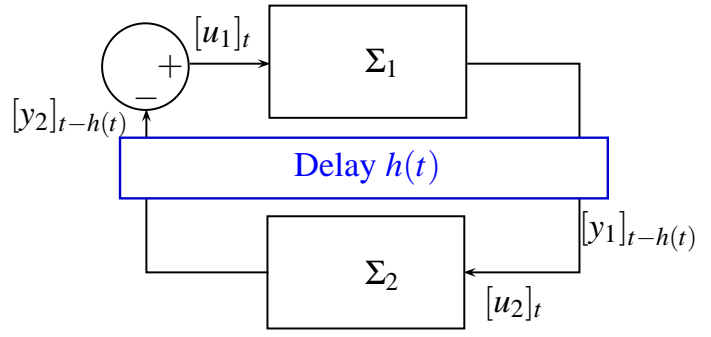

Fig. 2. Feedback interconnection of two PHSs with time-varying delay.

as it is shown in Fig 2. In (7), $h(t)$ represents the time-varying delay, which is assumed to satisfy the following conditions

$$
0 \leq h(t) \leq h_{M} \quad \text { and } \quad 0 \leq \dot{h}(t) \leq d<1,
$$

where $h_{M}$ is the maximum delay. By using the delayed interconnection (7), the interconnected port-Hamiltonian system takes the following form:

$$
\left\{\begin{aligned}
\frac{\mathrm{d}}{\mathrm{d} t}\left[\begin{array}{l}
x_{1} \\
x_{2}
\end{array}\right]= & {\left[\begin{array}{cc}
J_{1}-R_{1} & 0 \\
0 & J_{2}-R_{2}
\end{array}\right]\left[\begin{array}{l}
{\left[\nabla_{x_{1}} H_{1}\right]_{t}} \\
{\left[\nabla_{x_{2}} H_{2}\right]_{t}}
\end{array}\right] } \\
& +\left[\begin{array}{cc}
0 & -G_{1} G_{2}^{T} \\
G_{2} G_{1}^{T} & 0
\end{array}\right]\left[\begin{array}{l}
{\left[\nabla_{x_{1}} H_{1}\right]_{t-h(t)}} \\
{\left[\nabla_{x_{2}} H_{2}\right]_{t-h(t)}}
\end{array}\right] \\
\Longleftrightarrow \quad \dot{\mathcal{X}}= & (\mathcal{J}-\mathcal{R}) \nabla_{\mathcal{X}} \mathcal{H}_{t}+\mathcal{M} \nabla_{\mathcal{X}} \mathcal{H}_{t-h(t)} .
\end{aligned}\right.
$$

The previous equation no longer preserves the portHamiltonian structure because of the time varying delay. Indeed the energy balance equation of (9) becomes:

$$
\frac{\mathrm{d} \mathcal{H}_{t}}{\mathrm{~d} t} \stackrel{(9)}{=}-\nabla_{\mathcal{X}} \mathcal{H}_{t}^{T} \mathcal{R} \nabla_{\mathcal{X}} \mathcal{H}_{t}+\nabla_{\mathcal{X}} \mathcal{H}_{t}^{T} \mathcal{M} \nabla_{\mathcal{X}} \mathcal{H}_{t-h(t)},
$$

and this relation clearly shows that energy balance of the total Hamiltonian $\mathcal{H}$ in the presence of time-varying delays is not sufficient to conclude on stability of the interconnected system (9). Actually the last term of (10) has to be taken into account in the stability analysis. Hence, our objective is the design of a new Hamiltonian, which must be chosen in the sense of Lyapunov-Krasovskii in order to account for the delay.

In contrast with the literature where stability conditions of (9) derives from Jensen's inequality [9], [15], the present work proposes some new sufficient delay-dependent robust stability conditions for interconnected PHSs based on Wirtinger's integral inequality [11]. The benefits of such research directions comes from the reduction of conservatism compared with the usual Jensen inequality. The Wirtinger's inequality is recalled in the following lemma.

Lemma 1: [11] For a given symmetric positive definite $n \times n$ matrix $\mathcal{Q}>0$ and any differentiable function $f:[a, b] \rightarrow$ $\mathbb{R}^{n}$, the following inequality holds

$$
\int_{a}^{b} \dot{f}(s)^{T} \mathcal{Q} \dot{f}(s) \mathrm{d} s \geq \frac{1}{b-a}\left[\begin{array}{l}
\Omega_{0} \\
\Omega_{1}
\end{array}\right]^{T}\left[\begin{array}{cc}
\mathcal{Q} & 0 \\
* & 3 \mathcal{Q}
\end{array}\right]\left[\begin{array}{l}
\Omega_{0} \\
\Omega_{1}
\end{array}\right]
$$

where

$$
\Omega_{0}=f(b)-f(a), \Omega_{1}=f(b)+f(a)-\frac{2}{b-a} \int_{a}^{b} f(s) \mathrm{d} s .
$$

Remark 1: As mentioned in [11], the authors clearly showed that the term $\frac{1}{b-a} \Omega_{0}^{T} \mathcal{Q} \Omega_{0}$ presented in Lemma 1 refers exactly to Jensen's inequality, and the second term $\frac{3}{b-a} \Omega_{1}^{T} \mathcal{Q} \Omega_{1}$ is non-negative definite. Thus it is clear that Wirtinger's inequality encompasses Jensen's inequality.

\section{MAIN RESUlT}

In this section, we consider the stability problem of the interconnected port-Hamiltonian system (9) with timevarying delay. By introducing the same Lyapunov-Krasovskii functions as [15] and using the Wirtinger's inequality instead of the Jensen's Inequality, we can establish the stability theorems provided in the following subsection.

\section{A. Delay-dependent stability analysis}

Theorem 1: The delayed interconnected port-Hamiltonian system (9) is asymptotically stable for any time-varying delay $h(t)$ satisfying (8), if there exist matrices $\mathcal{P}>0, \mathcal{S}>0$ and $\mathcal{Q}>0$ of appropriate dimensions such that the following LMI holds:

$$
\Xi=\left[\begin{array}{ccc}
\Xi_{11} & \Xi_{12} & \Xi_{13} \\
* & \Xi_{22} & \Xi_{23} \\
* & * & \Xi_{33}
\end{array}\right]<0
$$

where

$$
\begin{aligned}
\Xi_{11}= & -\mathcal{R}+\mathcal{S}-4 \mathcal{Q}+(\mathcal{J}-\mathcal{R})^{T} \alpha(\mathcal{J}-\mathcal{R}) \\
& \quad+\mathcal{P} \nabla_{\mathcal{X}}^{2} \mathcal{H}_{t}(\mathcal{J}-\mathcal{R})+(\mathcal{J}-\mathcal{R})^{T} \nabla_{\mathcal{X}}^{2} \mathcal{H}_{t}^{T} \mathcal{P} \\
\Xi_{12}= & \frac{1}{2} \mathcal{M}-2 \mathcal{Q}+\mathcal{P} \nabla_{\mathcal{X}}^{2} \mathcal{H}_{t} \mathcal{M}+(\mathcal{J}-\mathcal{R})^{T} \alpha \mathcal{M} \\
\Xi_{13}= & 6 \mathcal{Q}, \quad \Xi_{23}=6 \mathcal{Q}, \quad \Xi_{33}=-12 \mathcal{Q} \\
\Xi_{22}= & -(1-d) \mathcal{S}-4 \mathcal{Q}+\mathcal{M}^{T} \alpha \mathcal{M} \\
\alpha= & h_{M}^{2} \nabla_{\mathcal{X}}^{2} \mathcal{H}_{t}^{T} \mathcal{Q} \nabla_{\mathcal{X}}^{2} \mathcal{H}_{t}
\end{aligned}
$$

Proof: The following Lyapunov-Krasovskii functional is chosen as candidate for the system (9):

$$
\mathcal{V}(t)=\mathcal{V}_{1}(t)+\mathcal{V}_{2}(t)+\mathcal{V}_{3}(t)
$$

where

$$
\begin{aligned}
& \mathcal{V}_{1}(t)=\mathcal{H}_{t}+\nabla_{\mathcal{X}} \mathcal{H}_{t}^{T} \mathcal{P} \nabla_{\mathcal{X}} \mathcal{H}_{t} \\
& \mathcal{V}_{2}(t)=\int_{t-h(t)}^{t} \nabla_{\mathcal{X}} \mathcal{H}_{s}^{T} \mathcal{S} \nabla_{\mathcal{X}} \mathcal{H}_{s} \mathrm{~d} s \\
& \mathcal{V}_{3}(t)=h_{M} \int_{-h_{M}}^{0} \int_{t+s}^{t} \frac{\mathrm{d}}{\mathrm{d} \tau}\left(\nabla_{\mathcal{X}} \mathcal{H}_{\tau}^{T}\right) \mathcal{Q} \frac{\mathrm{d}}{\mathrm{d} \tau}\left(\nabla_{\mathcal{X}} \mathcal{H}_{\tau}\right) \mathrm{d} \tau \mathrm{d} s
\end{aligned}
$$

Along the trajectories of (9), the derivative of the function $\mathcal{V}_{1}$ is given by:

$$
\begin{aligned}
\dot{\mathcal{V}}_{1}(t)= & -\nabla_{\mathcal{X}} \mathcal{H}_{t}^{T} \mathcal{R} \nabla_{\mathcal{X}} \mathcal{H}_{t}+\nabla_{\mathcal{X}} \mathcal{H}_{t}^{T} \mathcal{M} \nabla_{\mathcal{X}} \mathcal{H}_{t-h(t)} \\
& +\nabla_{\mathcal{X}} \mathcal{H}_{t}^{T} \mathcal{P} \nabla_{\mathcal{X}}^{2} \mathcal{H}_{t}^{T} \dot{\mathcal{X}}_{t}+\dot{\mathcal{X}}_{t}^{T} \nabla_{\mathcal{X}}^{2} \mathcal{H}_{t} \mathcal{P}^{T} \nabla_{\mathcal{X}} \mathcal{H}_{t} \\
= & \nabla_{\mathcal{X}} \mathcal{H}_{t}^{T}\left\{-\mathcal{R}+\mathcal{P} \nabla_{\mathcal{X}}^{2} \mathcal{H}_{t}(\mathcal{J}-\mathcal{R})\right. \\
& \left.+(\mathcal{J}-\mathcal{R})^{T} \nabla_{\mathcal{X}}^{2} \mathcal{H}_{t}^{T} \mathcal{P}^{T}\right\} \nabla_{\mathcal{X}} \mathcal{H}_{t} \\
& +\nabla_{\mathcal{X}} \mathcal{H}_{t}^{T}\left\{\frac{1}{2} \mathcal{M}+\mathcal{P} \nabla_{\mathcal{X}}^{2} \mathcal{H}_{t} \mathcal{M}\right\} \nabla_{\mathcal{X}} \mathcal{H}_{t-h(t)} \\
& +\nabla_{\mathcal{X}} \mathcal{H}_{t-h(t)}^{T}\left\{\frac{1}{2} \mathcal{M}^{T}+\mathcal{M}^{T} \nabla_{\mathcal{X}}^{2} \mathcal{H}_{t}^{T} \mathcal{P}^{T}\right\} \nabla_{\mathcal{X}} \mathcal{H}_{t} .
\end{aligned}
$$


The time derivative of $\mathcal{V}_{2}$ leads to:

$$
\begin{aligned}
\dot{\mathcal{V}}_{2}(t) & =\nabla_{\mathcal{X}} \mathcal{H}_{t}^{T} \mathcal{S} \nabla_{\mathcal{X}} \mathcal{H}_{t}-(1-\dot{h}(t)) \nabla_{\mathcal{X}} \mathcal{H}_{t-h(t)}^{T} \mathcal{S} \nabla_{\mathcal{X}} \mathcal{H}_{t-h(t)} \\
& \leq \nabla_{\mathcal{X}} \mathcal{H}_{t}^{T} \mathcal{S} \nabla_{\mathcal{X}} \mathcal{H}_{t}-(1-d) \nabla_{\mathcal{X}} \mathcal{H}_{t-h(t)}^{T} \mathcal{S} \nabla_{\mathcal{X}} \mathcal{H}_{t-h(t)}
\end{aligned}
$$

Finally, the time derivative of $\mathcal{V}_{3}$ is :

$$
\begin{aligned}
\dot{\mathcal{V}}_{3}(t)= & \dot{\mathcal{X}} \nabla_{\mathcal{X}}^{2} \mathcal{H}_{t}^{T} h_{M}^{2} \mathcal{Q} \nabla_{\mathcal{X}}^{2} \mathcal{H}_{t} \dot{\mathcal{X}} \\
& -h_{M} \int_{t-h_{M}}^{t} \frac{\mathrm{d}}{\mathrm{d} t}\left(\nabla_{\mathcal{X}} \mathcal{H}_{s}^{T}\right) \mathcal{Q} \frac{\mathrm{d}}{\mathrm{d} t}\left(\nabla_{\mathcal{X}} \mathcal{H}_{s}\right) \mathrm{d} s \\
= & \left\{\nabla_{\mathcal{X}} \mathcal{H}_{t}^{T}(\mathcal{J}-\mathcal{R})^{T}+\nabla_{\mathcal{X}} \mathcal{H}_{t-h(t)}^{T} \mathcal{M}^{T}\right\} \times \\
\alpha & \times\left\{(\mathcal{J}-\mathcal{R}) \nabla_{\mathcal{X}} \mathcal{H}_{t}+\mathcal{M} \nabla_{\mathcal{X}} \mathcal{H}_{t-h(t)}\right\} \\
& -h_{M} \int_{t-h_{M}}^{t} \frac{\mathrm{d}}{\mathrm{d} t}\left(\nabla_{\mathcal{X}} \mathcal{H}_{s}^{T}\right) \mathcal{Q} \frac{\mathrm{d}}{\mathrm{d} t}\left(\nabla_{\mathcal{X}} \mathcal{H}_{s}\right) \mathrm{d} s,
\end{aligned}
$$

where the parameter $\alpha=h_{M}^{2} \nabla_{\mathcal{X}}^{2} \mathcal{H}_{t}^{T} \mathcal{Q} \nabla_{\mathcal{X}}^{2} \mathcal{H}_{t}$.

The next step consists in applying the Wirtinger-based integral inequality stated in Lemma 1 . The last term of the the previous equation satisfies the following inequality

$$
\begin{aligned}
-h_{M} \int_{t-h_{M}}^{t} \frac{\mathrm{d}}{\mathrm{d} t}\left(\nabla_{\mathcal{X}} \mathcal{H}_{s}^{T}\right) \mathcal{Q} \frac{\mathrm{d}}{\mathrm{d} t}\left(\nabla_{\mathcal{X}} \mathcal{H}_{s}\right) \mathrm{d} s \leq \\
-\xi^{T}(t)\left[\begin{array}{cc}
\mathcal{Q} & 0 \\
* & 3 \mathcal{Q}
\end{array}\right] \xi(t),
\end{aligned}
$$

where the augmented vector $\xi(t)$ is given by

$$
\xi(t)=\left[\begin{array}{c}
\nabla_{\mathcal{X}} \mathcal{H}_{t}-\nabla_{\mathcal{X}} \mathcal{H}_{t-h(t)} \\
\left.\nabla_{\mathcal{X}} \mathcal{H}_{t}+\nabla_{\mathcal{X}} \mathcal{H}_{t-h(t)}-\frac{2}{h(t)} \int_{t-h(t)}^{t} \nabla_{\mathcal{X}} \mathcal{H}_{s} \mathrm{~d} s\right]
\end{array}\right]
$$

Thanks to this definition, the derivative of the LyapunovKrasovskii term $\mathcal{V}_{3}$ can be upper bounded as follows:

$$
\begin{aligned}
\dot{\mathcal{V}}_{3}(t)= & \left\{\nabla_{\mathcal{X}} \mathcal{H}_{t}^{T}(\mathcal{J}-\mathcal{R})^{T}+\nabla_{\mathcal{X}} \mathcal{H}_{t-h(t)}^{T} \mathcal{M}^{T}\right\} \times \\
& \alpha \times\left\{(\mathcal{J}-\mathcal{R}) \nabla_{\mathcal{X}} \mathcal{H}_{t}+\mathcal{M} \nabla_{\mathcal{X}} \mathcal{H}_{t-h(t)}\right\} \\
& -\xi^{T}(t)\left[\begin{array}{cc}
\mathcal{Q} & 0 \\
* & 3 \mathcal{Q}
\end{array}\right] \xi(t) .
\end{aligned}
$$

Combining (17), (18) and the inequality (20), the time derivative of the global energy (13) can be upper bounded as follows:

$$
\dot{\mathcal{V}}(t)=\left[\begin{array}{c}
\nabla_{\mathcal{X}} \mathcal{H}_{t} \\
\nabla_{\mathcal{X}} \mathcal{H}_{t-h(t)} \\
\frac{1}{h(t)} \int_{t-h(t)}^{t} \nabla_{\mathcal{X}} \mathcal{H}_{s} \mathrm{~d} s
\end{array}\right]^{T} \Xi\left[\begin{array}{c}
\nabla_{\mathcal{X}} \mathcal{H}_{t} \\
\nabla_{\mathcal{X}} \mathcal{H}_{t-h(t)} \\
\frac{1}{h(t)} \int_{t-h(t)}^{t} \nabla_{\mathcal{X}} \mathcal{H}_{s} \mathrm{~d} s
\end{array}\right]<0
$$

where the matrix $\Xi$ is given in (12).

Consequently, if there exists a solution to the LMI problem (12), then the interconnected port-Hamiltonian system (9) is asymptotically stable for all time varying delay $h(t)$, which satisfies condition (8).

\section{B. Robust delay-dependent stability analysis}

This section aims at extending the stability conditions from Theorem 1 to the case of robust stability. Indeed, one may consider interconnected port-Hamiltonian systems (9) subject to uncertainties in the model. Indeed the Hessian matrix $\nabla_{\mathcal{X}}^{2} \mathcal{H}$ in (9) might be uncertain in some application. In the following we will assume that the uncertainties are embedded in some polytopic representation, which are expressed as follows:

$$
\nabla_{\mathcal{X}}^{2} \mathcal{H}_{t}=\sum_{j=1}^{N} \lambda_{j}\left(\nabla_{\mathcal{X}}^{2} \mathcal{H}_{t}\right)^{(j)}
$$

where

$$
\sum_{j=1}^{N} \lambda_{j}=1, \quad 0 \leq \lambda_{j} \leq 1, \quad j=1,2, \ldots, N
$$

The matrices $\left(\nabla_{\mathcal{X}}^{2} \mathcal{H}_{t}\right)^{(j)}$ represent the vertices of the polytope which contains the Hessian and are assumed to be known. These matrices may also depend on the state variables, as it will be shown in the numerical example. In such a situation, the following result holds:

Theorem 2: The delayed interconnected port-Hamiltonian system (9) with polytopic uncertainties (22) is robustly asymptotically stable for any time-varying delay $h(t)$ satisfying (8), if there exist matrices $\mathcal{P}>0, \mathcal{S}>0$ and $\mathcal{Q}>0$ with appropriated dimensions, such that the following LMI holds:

$$
\Xi^{(j)}=\left[\begin{array}{ccc}
\Xi_{11}^{(j)} & \Xi_{12}^{(j)} & \Xi_{13} \\
* & \Xi_{22}^{(j)} & \Xi_{23} \\
* & * & \Xi_{33}
\end{array}\right]<0, \text { for } j=1, \ldots, N .
$$

where

$$
\begin{gathered}
\Xi_{11}^{(j)}=- \\
-\mathcal{R}+\mathcal{S}+(\mathcal{J}-\mathcal{R})^{T} \alpha^{(j)}(\mathcal{J}-\mathcal{R}) \\
-4 \mathcal{Q}+\mathcal{P}\left(\nabla_{\mathcal{X}}^{2} \mathcal{H}_{t}\right)^{(j)}(\mathcal{J}-\mathcal{R}) \\
+(\mathcal{J}-\mathcal{R})^{T}\left(\nabla_{\mathcal{X}}^{2} \mathcal{H}_{t}^{T}\right)^{(j)} \mathcal{P} \\
\Xi_{12}^{(j)}=\frac{1}{2} \mathcal{M}-2 \mathcal{Q}+\mathcal{P}\left(\nabla_{\mathcal{X}}^{2} \mathcal{H}_{t}\right)^{(j)} \mathcal{M} \\
+(\mathcal{J}-\mathcal{R})^{T} \alpha^{(j)} \mathcal{M} \\
\Xi_{13}=6 \mathcal{Q}, \quad \Xi_{23}=6 \mathcal{Q}, \quad \Xi_{33}=-12 \mathcal{Q} \\
\Xi_{22}^{(j)}=-(1-d) \mathcal{S}-4 \mathcal{Q}+\mathcal{M}^{T} \alpha^{(j)} \mathcal{M} \\
\alpha^{(j)}=h_{M}^{2}\left(\nabla_{\mathcal{X}}^{2} \mathcal{H}_{t}^{T}\right)^{(j)} \mathcal{Q}\left(\nabla_{\mathcal{X}}^{2} \mathcal{H}_{t}\right)^{(j)}
\end{gathered}
$$

Proof: The proof of this theorem follows the one of Theorem 1 and uses, in addition, the Schur complement to the term depending on $\alpha$ in order to prove convexity with respect to the polytopic uncertainties.

\section{Discussion}

As a by-product of Theorem 1, the constant delay case is derived. Consider an integer $h>0$ such that $h(t)=h$ for any time $t$. It follows 
Corollary 1: The time-constant delay interconnected system (9) is asymptotically stable if there exists the real matrices $\mathcal{S}=\mathcal{S}^{T}>0$ and $\mathcal{P}=\mathcal{P}^{T}>0$ in $\mathbb{R}^{n \times n}$ such that:

$$
\Gamma=\left[\begin{array}{cc}
\Gamma_{11} & \Gamma_{12} \\
* & \Gamma_{22}
\end{array}\right]<0
$$

where

$$
\begin{aligned}
& \Gamma_{11}=-\mathcal{R}+\mathcal{S}+\mathcal{P} \nabla_{\mathcal{X}}^{2} \mathcal{H}_{t}(\mathcal{J}-\mathcal{R})+(\mathcal{J}-\mathcal{R})^{T} \nabla_{\mathcal{X}}^{2} \mathcal{H}_{t}^{T} \mathcal{P} \\
& \Gamma_{12}=\frac{1}{2} \mathcal{M}+\mathcal{P} \nabla_{\mathcal{X}}^{2} \mathcal{H}_{t} \mathcal{M} \\
& \Gamma_{22}=-\mathcal{S}
\end{aligned}
$$

This stability condition is delay-independent and the proof follows from the previous theorem. This result can be easily checked if we take the functions $\mathcal{V}_{1}(t)$ and $\mathcal{V}_{2}(t)$ in (13) as Lyapunov-Krasovskii candidates.

Note that, due to the conservatism of this method, the ideal case $h(t) \equiv 0$ cannot be directly recovered from (12). However, it can be seen that $\mathcal{V}_{2}(t) \equiv 0, \mathcal{V}_{3}(t) \equiv 0$ and the interconnected dynamics (9) can be reduced to the delayfree case, which is stable, by using the Lyapounov function $\mathcal{V}(t)=\mathcal{V}_{1}(t)$ (with $\left.\mathcal{P}=0\right)$, which corresponds to the global energy of the system.

The stability condition proposed in (12) can be extended to other interconnections, such as feedback interconnections with two different delays. This can be motivated by the fact that the time that information takes to achieve its destination is different "on each side" with respect to the controller. This will not be presented here but in further researches.

Another important case to be discussed is the linear portHamiltonian system one, when $\mathcal{H}$ has the quadratic form $\mathcal{H}=\frac{1}{2} \mathcal{X}^{T} \mathcal{Q} \mathcal{X}$. It is important to note that the stability condition of the delayed interconnection remains the same as (12) by replacing the gradient with:

$$
\nabla_{\mathcal{X}} \mathcal{H}_{t}=\mathcal{Q} \mathcal{X}_{t}, \quad \mathcal{Q}=\mathcal{Q}^{T}>0 .
$$

\section{EXAMPLE}

Let us consider now an example corresponding to a damped pendulum. Its dynamics is described by a portHamiltonian system

$$
\left[\begin{array}{c}
\dot{q} \\
\dot{p}
\end{array}\right]=\left[\begin{array}{cc}
0 & 1 \\
-1 & -D
\end{array}\right]\left[\begin{array}{c}
\sin (q) \\
p
\end{array}\right]+\left[\begin{array}{l}
0 \\
1
\end{array}\right] u \text { and } y=p,
$$

with energy function

$$
H(q, p)=\frac{1}{2} p^{2}+(1-\cos (q)),
$$

where $D=0.1$ is the positive constant damping. Note that $(0,0)$ is an equilibrium of (27).

We consider a controller in port-Hamiltonian form given by

$$
\dot{\xi}=u_{c} \quad \text { and } \quad y_{c}=\nabla_{\xi} H_{c}
$$

with energy $H_{c}$ to be designed. The system and the controller are interconnected in a power-preserving way $u=-y_{c}$ and $u_{c}=y$ (feedback interconnection as in Fig 1), leading to the port-Hamiltonian closed-loop dynamics

$$
\left[\begin{array}{c}
\dot{q} \\
\dot{p} \\
\dot{\xi}
\end{array}\right]=\left[\begin{array}{ccc}
0 & 1 & 0 \\
-1 & -D & -1 \\
0 & 1 & 0
\end{array}\right]\left[\begin{array}{c}
\sin (q) \\
p \\
\nabla_{\xi} H_{c}
\end{array}\right]
$$

The control issue is to stabilize the closed-loop system at the point $\left(q_{*}, 0, \xi_{*}\right)$. The idea is to make use of a dynamical invariant called Casimir function and to construct a Lyapunov function based on the interconnected energy and Casimir function as follows

$$
H_{d}(q, p, \xi)=H(q, p)+H_{c}(\xi)+C(q, p, \xi) .
$$

This methodology and this example in particular has been extensively reported in [3], where it has been shown that the desired stability objectives are obtained by the following choices of controller Hamiltonian and Casimir function:

$$
\begin{aligned}
& H_{c}(\xi)=\frac{1}{2} \beta\left(\xi-\xi_{*}-\frac{1}{\beta} \sin (q)\right)^{2}, \\
& C(q, p, \xi)=\frac{1}{2} \kappa\left(q-q_{*}-\left(\xi-\xi_{*}\right)-\frac{1}{\kappa} \sin \left(q_{*}\right)\right)^{2},
\end{aligned}
$$

where $\beta$ and $\kappa$ are constant parameters satisfying

$$
\cos \left(q_{*}\right)+\kappa>0 \text { and } \beta \cos \left(q_{*}\right)+\kappa \cos \left(q_{*}\right)+\beta \kappa>0 .
$$

We further add damping in the system of the form $\dot{\xi}=$ $-z \nabla_{\xi} H_{c}$ with $z>0$.

Now assume that there is a delay in communication between the plant and the controller which satisfies (8). The interconnected system with delay then writes

$$
\begin{gathered}
{\left[\begin{array}{c}
\dot{q} \\
\dot{p} \\
\dot{\xi}
\end{array}\right]=\left[\begin{array}{ccc}
0 & 0 & 1 \\
-1 & -D & 0 \\
0 & 0 & -z
\end{array}\right]\left[\begin{array}{l}
{\left[\nabla_{q} H_{d}\right]_{t}} \\
{\left[\nabla_{p} H_{d}\right]_{t}} \\
{\left[\nabla_{\xi} H_{d}\right]_{t}}
\end{array}\right]} \\
+\left[\begin{array}{ccc}
0 & 0 & 0 \\
0 & 0 & -1 \\
0 & 1 & 0
\end{array}\right]\left[\begin{array}{l}
{\left[\nabla_{q} H_{d}\right]_{t-h(t)}} \\
{\left[\nabla_{p} H_{d}\right]_{t-h(t)}} \\
{\left[\nabla_{\xi} H_{d}\right]_{t-h(t)}}
\end{array}\right] .
\end{gathered}
$$

The stability condition also requires computing the Hessian of the Lyapunov function of the system without delay, given by [9]:

$$
\nabla^{2} H_{d}(q, p, \xi)=\left[\begin{array}{ccc}
\cos (q)+K & 0 & -K \\
0 & 1 & 0 \\
-K & 0 & \beta+K
\end{array}\right] .
$$

In order to satisfy the LMI (12) for the (delay-free) system we choose $K=2$ and $\beta=3$. We further choose $\kappa=z=1$.

In this example, the Hessian matrix (34) depends on the nonlinear term $\cos (q)$ which can only take values in the interval $[-1,1]$. Theorem 2, ensures stability of the delayed pendulum system (33) for several values of the upper bounds of the delay function and of its time derivative. A comparison between the stability conditions provided in [9] and the ones provided in the present article are reported in Table I. From Table I, one can see that the sufficient conditions for the stability problem proposed in this paper is less conservative than the results in [9]. Note that the stability conditions derived in [15] cannot be satisfied for this example. 


\begin{tabular}{|c||c|c|c|c|c|c|c|}
\hline$d$ & 0 & 0.2 & 0.4 & 0.6 & 0.8 & 0.9 & 0.99 \\
\hline \hline$[9]$ & 0.052 & 0.044 & 0.035 & 0.026 & 0.015 & 0.008 & - \\
\hline Theo.1 & 0.361 & 0.337 & 0.315 & 0.292 & 0.269 & 0.258 & 0.248 \\
\hline
\end{tabular}

TABLE I

THE MAXIMAL DELAYS $h_{m}$ FOR VARIOUS VALUES OF $d \in[0,1)$ FOR THE DELAYED PENDULUM (33).

\section{CONCLUSION}

This article provides a novel stability analysis for delayed port-Hamiltonian systems. The novelty of the approach relies on the use of the Wirtinger-based integral inequality. Sufficient stability conditions are derived in terms of a feasibility problem of an LMI constraint. Robust stability conditions have also been established for delayed interconnected portHamiltonian system with polytopic-type uncertainties. The advantage of the proposed method lies in the reduction of the conservatism with respect to the existing solutions from the literature, as we have illustrated in our numerical example.

\section{REFERENCES}

[1] E. Fridman and I. S. Niculescu. On complete Lyapunov-Krasovskii functional techniques for uncertain systems with fast-varying delays. International Journal of Robust and Nonlinear Control, 18(3):364374, 2008.

[2] E. Fridman and U. Shaked. An improved stabilization method for linear time-delay systems. IEEE Transactions on Automatic Control, 47(11):1931-1937, 2002.

[3] E. Garcia-Canseco, R. Pasumarthy, A.J. van der Schaft, and R. Ortega. On control by interconnection of port-Hamiltonian systems. In P. Piztek, editor, 16th IFAC World Congress. Elsevier, July 2005.
[4] C. Y. Kao and R. Pasumarthy. Stability analysis of interconnected Hamiltonian systems under time delays. IET Control Theory and Applications, 6(4):570-577, 2012.

[5] J-H. Kim. Delay and its time-derivative dependent robust stability of time-delayed linear systems with uncertainty. Automatic Control, IEEE Transactions on, 46(5):789-792, May 2001.

[6] T. Mori. Criteria for asymptotic stability of linear time-delay systems. Automatic Control, IEEE Transactions on, 30(2):158-161, February 1985.

[7] S. I. Niculescu. Delay Effects on Stability: A Robust Control Approach. Springer, Heidelberg, LNCIS, 2001.

[8] S. I. Niculescu, E. Verriest, L. Dugard, and J. M Dion. Stability and robust stability of time-delay systems: A guided tour. Stability and control of time-delay systems, pages 1-71, 1998.

[9] R. Pasumarthy and C. Y. Kao. On stability of time delay Hamiltonian systems. In Americain Control Conference, pages 4909-4914, 2009.

[10] J. P. Richard. Time-delay systems:an overview of some recent advances and open problems. Automatica, 39(10):1667-1694, 2003.

[11] A. Seuret and F. Gouaisbaut. Wirtinger-based integral inequality: Aplacation to time-delay systems. Automatica, 49:2860-2866, 2013.

[12] A.J. van der Schaft. $L_{2}-$ gain and passivity techniques in nonlinear control. Springer Series in Comp. Math. 31, 1999.

[13] M. Wu, Y. He, J-H. She, and G-P. Liu. Delay-dependent criteria for robust stability of time-varying delay systems. Automatica, 40:1435 1439, 2004.

[14] R. Yang and Y. Wang. Stability analysis for a class of nonlinear time-delay systems via Hamiltonian functional method. In Intelligent Control and Automation (WCICA), 2010 8th World Congress on, 2010.

[15] Y.J. Yoo, J.H. Koo, and S.C. Won. Delay dependent stability condition for the port-Hamiltonian systems with time varying delay. In Control Conference (ASCC), 2011 8th Asian, pages 834-838, May 2011. 